\title{
EVALUATION OF QUALITY OF DRINKING WATER FROM BAGHDAD, IRAQ
}

\author{
*BARBOOTI, M. Mํ.;; BOLZONI, G².; MIRZA, I. A ${ }^{3}$..; PELOSI M². \\ BARILLI, L2.; KADHUM, $\mathrm{R}^{3}$.; \& PETERLONGO, $\mathrm{G}^{2}$. \\ ${ }^{1}$ School of Applied Sciences, University of Technology, Baghdad, \\ Iraq. \\ 2Water and Sanitation Team for the Emergencies - Italian Red \\ Cross Macaque Laboratories - Milan - Italy \\ ${ }^{3}$ Environmental Analysis Department, Ministry of Environment, \\ Baghdad, Iraq.
}

\author{
+Present Address: Department of Earth and Environmental Studies, \\ Montclair State University, Normal Avenue 1, \\ Montclair, NJ 07043, USA. \\ *aldhaherim@mail.montclair.edu
}

\begin{abstract}
This is a joint work between the Italian Red Cross and the Environmental Laboratories, Baghdad. The drinking water (DW) samples from 16 residential districts in Baghdad were chemically evaluated with reference to the raw water samples and water directly taken from the purification plants. In addition to the routinely measured parameters, 17 metals and 11 trihalomathane (THM) were measured. Generally, the samples of water analysed can be considered of good quality. The relatively high sulphate and aluminium contents results from the use of aluminium sulphate as flocculent. The ammonia and Nitrite concentrations were lower than the detectable limit, because ammonia is converted into chloramines and nitrite is converted into Nitrate during chlorination. This indicates no sewage contamination of the drinking water. The high chloride contents can be referred to the use of partially degraded hypo for the disinfection. The presence of THM's in the samples analysed is indicative of good disinfection process. The presence of these compounds is preferred better than bacterial contamination. The relatively high levels of zinc and iron have no impact on the quality of DW. Iron, however, was efficiently removed during the treatment, together with Manganese. Reference was done to the EU specification of drinking water regarding total hardness, chloride contents, sulphate, iron and THM's. As for the iron content, the original pH of the river water (7.5 and 8.0) ensures that Iron should not be present in soluble form at a detectable level. Corrosion of the pipes could be one of the reasons for the presence of iron.
\end{abstract}

Key Words: Drinking water quality, heavy metals, sulphate, Aluminium, Trihalomethans, hardness.

\section{INTRODUCTION}

Many techniques are used to remove pollutants, suspended matter and microbial agents from the water body. The regulations controlling the acceptable levels of water quality parameters are always updated to fulfill health standards. The monitoring of drinking water (DW) quality is receiving increasing interest and analytical methods are developed to improve the sensitivity and detection limits for various analytes. However, DW, including bottled water, may reasonably be expected to contain at least small amounts of some contaminants. The presence of contaminants does not necessarily indicate that water poses a health risk.
The DW is taken from rivers, lakes, reservoir, spring and wells. Along the trip of water over the surface land, some components of rocks and soil may dissolve and carried to the final consumer. Organic and inorganic contaminants may be introduced to DW sources from industrial processes, petroleum production and refining and domestic activities. The production of DW involves processes that remove suspended matter disinfection to remove biological agents. The appearance, taste or odors of water from a well or other sources offer some information on obvious contamination but chemical analysis is needed to detect water contamination. Obvious contaminants include silt (turbidity) and hydrogen sulfide, which can be detected by smell (Self, 1996). In some communities, the quality parameter levels of natural waters fall within the permissible limits that no treatment was necessary to use it as DW (Akoto \& Adiyiah, 2007).

Disinfection is an important and necessary step in the water treatment process to protect against harmful bacteria and other possible contamination. Chlorine is the most widely used and approved disinfectant in Iraq and most countries including the United States. Disinfection of water with chlorine introduced a major category of contaminants, the trihalomethanes, as a result of the action of chlorine on the organic contaminants (WHO, 2005, Singer, 2006; Tanglewood Water System, 2007).

When the DW standards are usually issued, they are used for governing drinking water quality and reporting requirements for public water systems (NSF International, 2000). Water contaminants may originate from the treatment chemicals, if their quality grades are not within the standards (Water Supply Division, 2005). Studies on DW quality monitoring reveal major gaps in the system of public health protections when it comes to tap water safety (Environmental Working Group, 2005) and to prevent water pollution and protect the sources of DW.

Vodela et al., (1997), studied the effects of contaminants like arsenic, cadmium, lead, benzene and trichloroethylene on reproductive performance, egg quality, and embryo toxicity in broiler breeders. They showed a linear relationship between increasing concentration of the chemical mixture in drinking water and decreasing body weight of hens. Low concentration of the chemical mixture significantly decreased egg production and egg weight, and increased percentage embryonic mortality. These results suggest that reproductive function in hens is sensitive to adverse effects of contaminated drinking water.

The pollution with toxic metals is a serious concern; because potential contamination of drinking water sources can originate from soil contamination. Some heavy metals have bio-importance as trace elements but, the biotoxic effects of many of them in human biochemistry are of great concern. Hence, there is the need 
for proper understanding of the conditions, such as the the concentrations and oxidation states, which make them harmful, and how biotoxicity occurs (Duruibe, 2007).

Squillqace, et al. studied the determination of volatile organic compound, VOCs, pesticides, Nitrate and their mixtures in groundwater used for drinking Water (Squillqace, 2002). Nitrate at levels above $45 \mathrm{mg} / \mathrm{L}$ is a health risk for infants of less than six months of age and pregnant women with certain specific enzyme deficiencies. Nitrate can interfere with the capacity of blood to carry oxygen (Squillqace, et al., 2002).

Fluoride has beneficial effects on teeth at low concentrations in DW, but excessive exposure to fluoride in drinking-water, can give rise to a number of adverse effects. These ranges from mild dental fluorosis to crippling skeletal fluorosis which is a significant cause of morbidity (Fawell, et al., 2006). The significance and health effects of some water pollutants and ingredients like sulfate, lead, fluoride, nitrate and organic compounds have been reported (Kendall, 1992).

In Iraq, the municipalities are responsible for the production and delivery of drinking water (DW). During 2004 and 2005 the quality of DW in Iraq was greatly deteriorated because of the war operation in this country. The analytical laboratories of the environment and water and health authorities carry out chemical and bacteriological test on DW in Iraq. The failure of the analyzed water samples reached about $40 \%$ of the collected samples Barbooti, et al., (2005). Some efforts were put to improve the infrastructure and reconstruction of DW treatment systems. Many projects were established in Iraq to supply bottled DW and manufacture of home water clarification, disinfection units in Iraq. A comprehensive study was carried out during 2006 for the evaluation of bottled DW and home purification units. Some bottled DW failed to match the minimum contaminant level (Barbooti, et al., 2006. Ion chromatography was used successfully for the determination of major anions, $\mathrm{PO}_{4}^{3-}, \mathrm{SO}_{4}{ }^{2-}, \mathrm{NO}_{3}{ }^{-}, \mathrm{NO}_{2}{ }^{-}, \mathrm{Cl}, \mathrm{Br}$ and $\mathrm{F}^{-}$in drinking water samples from Baghdad area and Kualalampur (Alsudani, et al., 2009).

Soylak et al., 2002, studied the determination of metal ions in the drinking water samples from Yozgat, Turkey. They concluded that the concentrations of the investigated major ions and metal ions in the drinking water samples were below the guidelines for DW standards (Water Pollution Control Regulation of Turkish Authorities, 1989 and WHO, 2004. No correlations were found between metal concentrations in the drinking water samples (Soylak et al., 2002).

It is the aim of the present work to make a comprehensive evaluation of the chemical parameters of the DW supplied to the homes in Baghdad area from the many water stations controlled by the Baghdad Amanat. The present work is a collaborative investigation on the levels of chemical pollutants in drinking water supply of Baghdad in cooperation with Esercizio Acque Reflue e Laboratori CAP Gestione spa Labratorio di Analisi - Milano - Italy.

\section{MATERIALS AND METHODS}

Sampling: Twenty seven water samples were collected for this study. Fifteen samples were taken from homes (Residence), 6 samples were freshly produced DW from the production stations and the remaining six were taken from the location of water intake from the river to these stations. The 15 residential locations represent both the eastern and western sides of Tigris River (Table 1). The locations of water production stations are displayed in Fig. 1.

\section{TABLE 1. NAMES OF LOCATIONS WHERE RESIDENTIAL SAMPLES WERE COLLECTED}

\begin{tabular}{l}
\hline Al Atifia 409/20/42 \\
\hline Al Jehad 889/28/31 \\
\hline Al Zoufrania 954/54/13 \\
\hline Al Mahmodia 14th Ramadan 22/7 \\
\hline Al Talbia 323/28/2 \\
\hline Al Mahmodia 17 \\
\hline Al Kadh July 69/106 \\
\hline Al Shaab 337/19/83 \\
\hline Al Amine 2end 741/36/15 \\
\hline Al Karada 903/12/5 \\
\hline Al Kadsia 602/8/50 \\
\hline Al Salehia 28 April building \\
\hline Palestine Street 503/34/19 \\
\hline Al Sadir 529/28/11 \\
\hline Al Kadsia 604/5/3
\end{tabular}

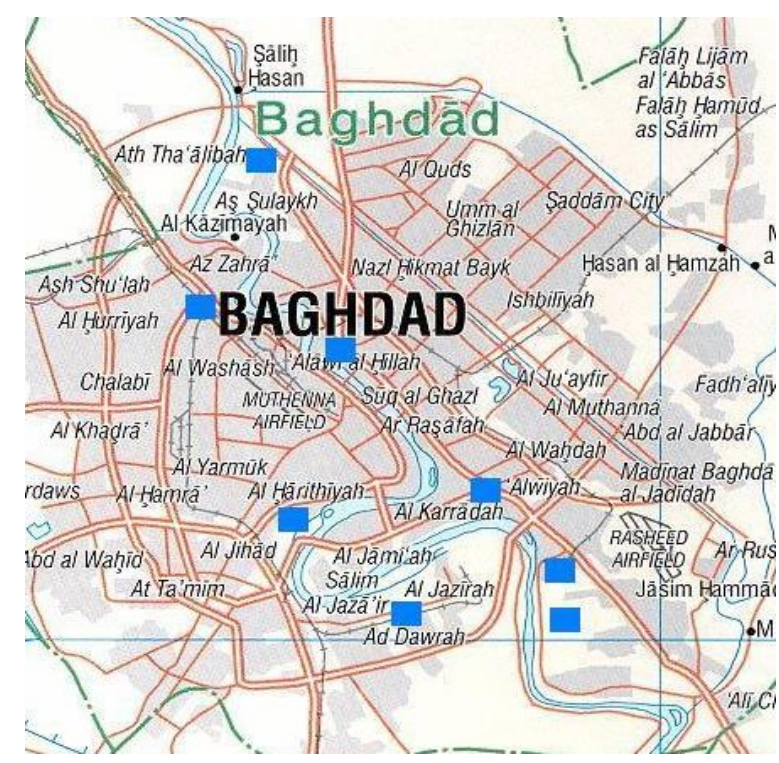

\section{FIG. 1. DISTRIBUTION OF THE DRINKING WATER (DW) PRODUCING STATIONS WITHIN BAGHDAD AREA.}

Induced Coupled Plasma analysis: The determination of metals was carried out using induce couple plasma optical emission spectroscopy (ICP-OES) on Varian/Vista-AX ICP-AES. High-purity nitric acid $\left(\mathrm{HNO}_{3} 60 \%\right.$, from Merck) was used. Deionized water (with $18 \mathrm{M} \Omega-\mathrm{cm}$ resistance) were used for dilution and preparation of standard solution. The calibration standards were prepared by diluting multi-element stock standards (Inorganic Ventures, Inc., 
Lakewood, $\mathrm{NJ}, \mathrm{USA}$ ) with $1 \% \mathrm{v} / \mathrm{v} \mathrm{HNO}_{3}$, and working standards of below $10 \mu \mathrm{g} / \mathrm{L}$ were prepared immediately before the measurement.

Ion Chromatography: Dionex DX-120 and DX-500 ion chromatography systems were used for this work. The system includes GP50 Gradient Pump, CD20 Conductivity Detector and AS40 Autosampler with 5-mL vials. The system was linked to A PeakNet Chromatography Workstation.

The eluent was $3.5 \mathrm{mM}$ sodium carbonate $/ 1.0 \mathrm{mM}$ sodium bicarbonate (IonPac AS4A). The sodium and potassium salts of the anions ACS grade, were used for the preparing of the standards. Deionized water (with $18 \mathrm{M} \Omega-\mathrm{cm}$ resistance) were used for dilution and preparation of standard solution.

IonPac AG4A-SC, $4 \times 50 \mathrm{~mm}$ column was used for the analysis of DW samples. The flow rate of the eluent was $2.0 \mathrm{~mL} / \mathrm{min}$. Sample aliquots of $50 \mu \mathrm{L}$ were injected and the detection was done with suppressed conductivity. The vial caps in the AS40 Automated Sampler contain a 20- $\mu \mathrm{m}$ filter, so no additional filtration was used in conjunction with this mode of sample introduction.

Gas Chromatography for THMs: A $10 \mathrm{~mL}$ aliquot of each water sample or standard was placed in $20 \mathrm{~mL}$ headspace autosampler vials which contained $2.5 \mathrm{~g}$ of $\mathrm{NaCl}$ to force the THMs gas phase where they are collected by the SPME fiber. The vials were incubated at $65^{\circ} \mathrm{C}$ for 30 minutes and agitated at $100 \mathrm{rpm}$ (10 second duration, every 60 seconds). Three minutes were necessary for the desorption at $260^{\circ} \mathrm{C}$. The analysis were performed on Agilent 6890 Gas Chromatograph with electron capture detector using heating program starting from $60^{\circ} \mathrm{C}(2$ minute hold), and heating up at $30^{\circ} \mathrm{C} /$ minute to $250^{\circ} \mathrm{C}$ (10 minute hold).

\section{RESULTS}

Table 2 shows the $\mathrm{pH}$, electrical conductivity (EC), total dissolved solids (TDS) and hardness of 15 residence samples and 12 water samples taken directly from the water production stations within Baghdad. The pH values fall between 7.05 and 7.84 and the average value was 7.67. The hardness values fall between 280 and $440 \mathrm{mg} / \mathrm{L}$ and the average is $339.6 \mathrm{mg} / \mathrm{L}$. The TDS values fall between 486 and $860 \mathrm{mg} / \mathrm{L}$ and the average is $546.3 \mathrm{mg} / \mathrm{L}$. The EC values fall between 673 and $1200 \mathrm{mg} / \mathrm{L}$ and the average is $827.0 \mathrm{mg} / \mathrm{L}$.

Table 3 shows the concentration values of the major anions: $\mathrm{SO}_{4}{ }^{2-}$ $\mathrm{Cl}^{-}, \mathrm{NO}_{3}^{-}$, and $\mathrm{F}^{-}$. The sulphate concentration values fall between 280 and $440 \mathrm{mg} / \mathrm{L}$ and the average is $230.1 \mathrm{mg} / \mathrm{L}$. The chloride concentration values fall between 50 and $120 \mathrm{mg} / \mathrm{L}$ and the average is $67.7 \mathrm{mg} / \mathrm{L}$.

TABLE 2. PHYSIOCHEMICAL CHARACTERISTICS OF TREATED (T) AND UNTREATED (R) WATER SAMPLES (DW) FROM BAGHDAD

\begin{tabular}{|c|c|c|c|c|c|}
\hline No. & Location & $\begin{array}{l}\text { Total } \\
\text { Hardness } \\
\text { (mg/L) }\end{array}$ & $\begin{array}{l}\text { TDS, mg/L } \\
(180)^{\circ} \mathrm{C} .\end{array}$ & $\begin{array}{l}\text { Conductivity } \\
\mu S / \mathrm{cm}\end{array}$ & $\mathrm{Ph}$ \\
\hline 1 & Utaifiya, Q409 & 410 & 725 & 1007 & 7.84 \\
\hline 2 & Jihad, Q889 & 280 & 501 & 695 & 7.74 \\
\hline 3 & Zufaraniya, Q954 & 430 & 770 & 1070 & 7.73 \\
\hline 4 & Mamudiya, Q14 Ramdan & 430 & 845 & 1173 & 7.71 \\
\hline 5 & Talbiya, Q 323 & 320 & 612 & 849 & 7.82 \\
\hline 6 & Mamudiya Q 17 july & 440 & 864 & 1200 & 7.73 \\
\hline 7 & Kadhmiya, Q402 & 420 & 749 & 1041 & 7.77 \\
\hline 8 & Shaab, Q337 & 310 & 608 & 843 & 7.84 \\
\hline 9 & I 2n Amin, 741 & 300 & 588 & 815 & 7.83 \\
\hline 10 & Qarrada, Q905 & 420 & 753 & 1046 & 7.75 \\
\hline 11 & Qarrada, Q905 & 350 & 642 & 892 & 7.74 \\
\hline 12 & Qadsiya, Q602 & 270 & 512 & 709 & 7.88 \\
\hline 13 & Salhiya, Q 28 Niss & 320 & 593 & 823 & 7.82 \\
\hline 14 & Falstin st, Q503 & 280 & 522 & 724 & 7.87 \\
\hline 15 & adr City, Q529 & 310 & 605 & 839 & 7.88 \\
\hline 16 & Dora Project $(\mathrm{R})$ & 320 & 492 & 681 & 7.05 \\
\hline 17 & Dora Project (T) & 310 & 487 & 675 & 7.28 \\
\hline 18 & Whda Project (R) & 310 & 486 & 673 & 7.51 \\
\hline 19 & Whda Project (T) & 310 & 494 & 685 & 7.56 \\
\hline 20 & 9 Apr Project (R) & 320 & 508 & 704 & 7.69 \\
\hline 21 & 9 Apr Project (T) & 320 & 507 & 703 & 7.81 \\
\hline 22 & Qadsiya Project (R) & 310 & 485 & 672 & 7.84 \\
\hline 23 & Qadsiya Project (T) & 310 & 489 & 677 & 7.79 \\
\hline 24 & Wathba Project(R) & 340 & 557 & 772 & 7.85 \\
\hline 25 & WathbaProject (T) & 350 & 574 & 796 & 7.33 \\
\hline 26 & Project karama(R) & 340 & 560 & 776 & 7.71 \\
\hline 27 & Karama Project (T) & 340 & 571 & 791 & 7.60 \\
\hline
\end{tabular}


TABLE 3. THE CONCENTRATION OF MAJOR ANIONS IN TREATED (T) AND UNTREATED (R) WATER SAMPLES (DW)

\begin{tabular}{|c|c|c|c|c|c|c|c|}
\hline No. & Location & $\begin{array}{l}\mathrm{Cl} \\
\mathrm{mg} / \mathrm{I}\end{array}$ & $\begin{array}{l}\mathrm{SO}_{4}^{2-} \\
\mathrm{mg} / \mathrm{I}\end{array}$ & $\begin{array}{l}\text { F- } \\
\mathrm{mg} / \mathrm{I}\end{array}$ & $\begin{array}{l}\mathrm{CSiO}_{2} \\
\mathrm{mg} / \mathrm{I}\end{array}$ & $\begin{array}{l}\text { Nitrite } \\
\text { (mg/L) }\end{array}$ & $\begin{array}{l}\text { Nitrate } \\
\text { (mg/L) }\end{array}$ \\
\hline & Iraqi Specification & 250 & 250 & 1.0 & & & \\
\hline & WHO Specification & 250 & 250 & 1.5 & & & \\
\hline & Europian Specification & 250 & 500 & 1.5 & & & \\
\hline 1 & Utaifiya, Q409 & 250 & 250 & 1.0 & - & $<0.025$ & 2 \\
\hline 2 & Jihad, Q889 & 250 & 250 & 1.5 & - & $<0.025$ & 2 \\
\hline 3 & Zufaraniya, Q954 & 250 & 500 & 1.5 & - & $<0.025$ & 2 \\
\hline 4 & Mamudiya, Q 14 Ramdn & 74 & 338 & $<0.5$ & 5 & $<0.025$ & $<1$ \\
\hline 5 & Talbiya, Q 323 & 50 & 173 & $<0.5$ & 5 & $<0.025$ & 2 \\
\hline 6 & Mamudiya Q 17 july & 77 & 370 & $<0.5$ & 5 & $<0.025$ & $<1$ \\
\hline 7 & Kadhmiya, Q402 & 120 & 386 & $<0.5$ & 5 & $<0.025$ & $<1$ \\
\hline 8 & Shaab, Q337 & 65 & 251 & $<0.5$ & 4 & $<0.025$ & 2 \\
\hline 9 & 2n Amin, 741 & 120 & 397 & $<0.5$ & 5 & $<0.025$ & 2 \\
\hline 10 & Qarrada, Q905 & 74 & 348 & $<0.5$ & 5 & $<0.025$ & 2 \\
\hline 11 & Qarrada, Q905 & 65 & 225 & $<0.5$ & 4 & $<0.025$ & 2 \\
\hline 12 & Qadsiya, Q602 & 61 & 200 & $<0.5$ & 5 & $<0.025$ & 2 \\
\hline 13 & Salhiya, Q 28 Niss & 72 & 336 & $<0.5$ & 5 & $<0.025$ & 2 \\
\hline 14 & Falstin st, Q503 & 62 & 257 & $<0.5$ & 5 & $<0.025$ & 2 \\
\hline 15 & Sadr City, Q529 & 49 & 162 & $<0.5$ & 4 & $<0.025$ & 2 \\
\hline 16 & Dora Project (R) & 55 & 215 & $<0.5$ & 4 & 1.49 & 4 \\
\hline 17 & Dora Project (T) & 50 & 168 & $<0.5$ & 4 & $<0.025$ & 6 \\
\hline 18 & Whda Project (R) & 64 & 230 & $<0.5$ & 4 & $<0.025$ & 6 \\
\hline 19 & Whda Project (T) & 57 & 159 & $<0.5$ & 8 & $<0.025$ & 6 \\
\hline 20 & 9 Apr Project (R) & 60 & 164 & $<0.5$ & 8 & $<0.025$ & 6 \\
\hline 21 & 9 Apr Project (T) & 56 & 158 & $<0.5$ & 10 & $<0.025$ & 6 \\
\hline 22 & Qadsiya Project (R) & 61 & 162 & $<0.5$ & 8 & $<0.025$ & 6 \\
\hline 23 & Qadsiya Project (T) & 63 & 167 & $<0.5$ & 10 & $<0.025$ & 6 \\
\hline 24 & Wathba Project(R) & 64 & 175 & $<0.5$ & 8 & $<0.025$ & 5 \\
\hline 25 & WathbaProject (T) & 56 & 157 & $<0.5$ & 9 & $<0.025$ & 6 \\
\hline 26 & Project karama(R) & 60 & 162 & $<0.5$ & 8 & $<0.025$ & 7 \\
\hline 27 & Karama Project (T) & 72 & 200 & $<0.5$ & 13 & $<0.025$ & 6 \\
\hline
\end{tabular}

The results of the major cations, $\mathrm{Ca}^{2+}, \mathrm{Na}^{+}, \mathrm{Mg}^{2+}$ and $\mathrm{K}^{+}$, concentrations are given in Table 4. The calcium concentration values fall between 61 and $118 \mathrm{mg} / \mathrm{L}$ and the average is 84.5 $\mathrm{mg} / \mathrm{L}$. The sodium concentration values fall between 20 and 32 $\mathrm{mg} / \mathrm{L}$ and the average is $24.3 \mathrm{mg} / \mathrm{L}$. The magnesium concentration values fall between 28 and $34 \mathrm{mg} / \mathrm{L}$ and the average is $31.1 \mathrm{mg} / \mathrm{L}$. The results of some common metal ions like iron, aluminium, zinc, ammonium and lithium are given in Table 5.

TABLE 4. THE CONCENTRATION OF THE COMMON CATIONS IN TREATED (TO AND RAW (R) WATER SAMPLES (DW) FROM BAGHDAD.

\begin{tabular}{lllllll}
\hline No. & Sample Location & $\begin{array}{l}\mathbf{C a}^{2+} \\
\mathbf{m g} / \mathbf{L}\end{array}$ & $\begin{array}{l}\mathbf{K}^{+} \\
\mathbf{m g} / \mathbf{L}\end{array}$ & $\begin{array}{l}\mathbf{M g}^{2+} \\
\mathbf{m g} / \mathbf{L}\end{array}$ & $\begin{array}{l}\mathbf{N a}^{+} \\
\mathbf{m g} / \mathbf{L}\end{array}$ & $\begin{array}{l}\text { Total } \\
\text { Cations } \\
\mathbf{m g} / \mathbf{L}\end{array}$ \\
\hline & Iraqi Specification & 50 & - & 50 & 200 & \\
& WHO Specification & - & - & - & - & \\
& Europian Specification & - & - & - & 200 & \\
1 & Utaifiya, Q 409 & 111 & 1 & 33 & 26 & 171 \\
2 & Jihad, Q889 & 62 & $<1$ & 29 & 21 & 113 \\
3 & Zufaraniya, Q954 & 118 & 2 & 32 & 26 & 178 \\
4 & Mamudiya, Q 14 Ramdn & 111 & $<1$ & 36 & 32 & 180 \\
5 & Talbiya, Q 323 & 68 & 1 & 35 & 26 & 148 \\
6 & Mamudiya Q 17 july & 118 & 1 & 36 & 25 & 180 \\
7 & Kadhmiya, Q402 & 116 & 1 & 32 & 24 & 173 \\
8 & Shaab, Q337 & 67 & 2 & 34 & 26 & 129 \\
9 & 2n Amin, 741 & 67 & 1 & 33 & 23 & 124 \\
10 & Qarrada, Q905 & 114 & $<1$ & 33 & 20 & 168 \\
11 & Qarrada, Q905 & 88 & 1 & 31 & 22 & 142 \\
12 & Qadsiya, Q602 & 61 & $<1$ & 29 & 21 & 112 \\
13 & Salhiya, Q 28 Nissan & 80 & 1 & 30 & 25 & 136 \\
\hline
\end{tabular}


TABLE 4 CONT. THE CONCENTRATION OF THE COMMON CATIONS IN TREATED (TO AND RAW (R) WATER SAMPLES (DW) FROM BAGHDAD.

\begin{tabular}{lllllll}
\hline 14 & Falstin st, Q503 & 63 & 1 & 30 & 25 & 119 \\
15 & Adr City, Q529 & 67 & 2 & 35 & 32 & 136 \\
16 & Dora Project (R) & 80 & 2 & 28 & 21 & 131 \\
17 & Dora Project (T) & 78 & 2 & 28 & 21 & 129 \\
18 & Whda Project (R) & 79 & 5 & 28 & 21 & 133 \\
19 & Whda Project (T) & 79 & 1 & 28 & 32 & 140 \\
20 & 9 Apr Project (R) & 79 & 2 & 29 & 22 & 132 \\
21 & 9 Apr Project (T) & 78 & $<1$ & 29 & 20 & 128 \\
22 & Qadsiya Project (R) & 78 & 2 & 28 & 21 & 129 \\
23 & Qadsiya Project (T) & 78 & 2 & 28 & 21 & 129 \\
24 & Wathba Project(R) & 84 & 2 & 31 & 26 & 143 \\
25 & WathbaProject (T) & 88 & 2 & 31 & 26 & 147 \\
26 & Project karama(R) & 83 & 2 & 32 & 26 & 143 \\
27 & Karama Project (T) & 86 & 2 & 31 & 26 & 145 \\
\hline
\end{tabular}

TABLE 5. THE CONCENTRATIONS OF IRON, ALUMINUM, LITHIUM, ZINC AND AMMONIUM IN TREATED (T) AND UNTREATED (R) WATER SAMPLES (DW).

\begin{tabular}{|c|c|c|c|c|c|c|}
\hline No. & Location & $\begin{array}{l}\mathrm{Fe} \\
\mu \mathrm{g} / \mathrm{L}\end{array}$ & $\begin{array}{l}\mathrm{Al} \\
\mathrm{mg} / \mathrm{L}\end{array}$ & $\begin{array}{l}\mathrm{Zn}, \\
\mu \mathrm{g} / \mathrm{L}\end{array}$ & $\begin{array}{l}\mathrm{NH}_{4}, \\
\mathrm{mg} / \mathrm{L}\end{array}$ & $\begin{array}{l}\mathrm{Li} \\
\mu \mathrm{g} / \mathrm{L}\end{array}$ \\
\hline & Iraqi Specification & 300 & 0.2 & 3000 & $<0.1$ & - \\
\hline & WHO Specification & - & 0.2 & - & $<0.1$ & - \\
\hline & Europian Specification & 200 & 0.2 & - & $<0.1$ & - \\
\hline 1 & Utaifiya, Q409 & 29 & 0.15 & 320 & $<0.1$ & $<1$ \\
\hline 2 & Jihad, Q889 & 76 & 0.18 & 84 & $<0.1$ & $<1$ \\
\hline 3 & Zufaraniya, Q954 & $<20$ & 0.13 & 77 & $<0.1$ & $<1$ \\
\hline 4 & Mamudia, Q 14 Ramdn & 29 & 0.06 & 427 & $<0.1$ & $<1$ \\
\hline 5 & Talbiya, Q 323 & $<20$ & 0.14 & 71 & $<0.1$ & $<1$ \\
\hline 6 & Mamudiya Q 17 july & $<20$ & 0.07 & 62 & $<0.1$ & $<1$ \\
\hline 7 & Kadhmiya, Q402 & $<20$ & 0.16 & 39 & $<0.1$ & $<1$ \\
\hline 8 & Shaab, Q337 & $<20$ & 0.14 & $<20$ & $<0.1$ & $<1$ \\
\hline 9 & 2n Amin, 741 & 47 & 0.18 & 26 & $<0.1$ & $<1$ \\
\hline 10 & Qarrada, Q905 & 52 & 0.11 & 280 & $<0.1$ & $<1$ \\
\hline 11 & Qarrada, Q905 & 59 & 0.08 & 963 & $<0.1$ & $<1$ \\
\hline 12 & Qadsiya, Q602 & 23 & 0.13 & 123 & $<0.1$ & $<1$ \\
\hline 13 & Salhiya, Q 28 Niss & $<20$ & 0.12 & 41 & $<0.1$ & $<1$ \\
\hline 14 & Falstin st, Q503 & 42 & 0.15 & 71 & $<0.1$ & $<1$ \\
\hline 15 & adr City, Q529 & $<20$ & 0.14 & 28 & $<0.1$ & $<1$ \\
\hline 16 & Dora Project (R) & 34 & 0.067 & 40 & $<0.1$ & $<1$ \\
\hline 17 & Dora Project (T) & $<20$ & 0.14 & $<20$ & $<0.1$ & $<1$ \\
\hline 18 & Whda Project $(\mathrm{R})$ & 233 & 0.33 & $<20$ & $<0.1$ & $<1$ \\
\hline 19 & Whda Project (T) & 32 & 0.17 & $<20$ & $<0.1$ & $<1$ \\
\hline 20 & 9 Apr Project (R) & 251 & 0.32 & $<20$ & $<0.1$ & $<1$ \\
\hline 21 & 9 Apr Project (T) & $<20$ & 0.12 & $<20$ & $<0.1$ & $<1$ \\
\hline 22 & Qadsiya Project (R) & 125 & 0.18 & $<20$ & $<0.1$ & $<1$ \\
\hline 23 & Qadsiya Project (T) & 31 & 0.14 & $<20$ & $<0.1$ & $<1$ \\
\hline 24 & Wathba Project(R) & 624 & 0.77 & $<20$ & $<0.1$ & $<1$ \\
\hline 25 & WathbaProject (T) & $<20$ & 0.07 & $<20$ & & $<1$ \\
\hline 26 & Project karama(R) & 595 & 0.73 & $<20$ & & $<1$ \\
\hline 27 & Karama Project (T) & $<20$ & 0.10 & $<20$ & & $<1$ \\
\hline
\end{tabular}

Table 6 shows the concentration values of trace and heavy metals in the DW and raw water samples. The concentration levels of trihalomethanes in the DW samples are listed in Table 7.

\section{DISCUSSION}

The $\mathrm{pH}$ values: The $\mathrm{pH}$ values of the water samples from the uptake stations (Prior to treatment) were between $7.05-7.85$ for
April 2006. For DW samples the $\mathrm{pH}$ values were in the range 7-28 -7.81 for April 2005. For the network the results indicated $\mathrm{pH}$ values of 7.74 to 7.88 for November 2005 and $7.51-7.96$ for DW samples of June 2005. All the results are within the acceptable limits $(6.5-8.5)$. However the WHO regulations do not include limits for the $\mathrm{pH}$ values for drinking water. European standard includes a range of $6.5-9.5$ for the $\mathrm{pH}$ of $\mathrm{DW}$. 


\section{TABLE 6. THE CONCENTRATION OF TRACE METALS IN TREATED (T) AND UNTREATED (R) WATER SAMPLES (DW)}

\begin{tabular}{|c|c|c|c|c|c|c|c|c|c|c|}
\hline No. & Location & $\begin{array}{l}\mathrm{Pb} \\
\mu \mathrm{g} / \mathrm{L}\end{array}$ & $\begin{array}{l}\text { B } \\
\mathrm{mg} / \mathrm{L}\end{array}$ & $\begin{array}{l}\mathrm{Ba} \\
\mathrm{mg} / \mathrm{L}\end{array}$ & $\begin{array}{l}\text { As } \\
\mu \mathrm{g} / \mathrm{L}\end{array}$ & $\begin{array}{l}\text { Cd } \\
\mu g / L\end{array}$ & $\begin{array}{l}\mathrm{Cu} \\
\mu \mathrm{g} / \mathrm{L}\end{array}$ & $\begin{array}{l}\mathrm{Ni} \\
\mu \mathrm{g} / \mathrm{L}\end{array}$ & $\begin{array}{l}\mathrm{Cr} \\
\mu \mathrm{g} / \mathrm{L}\end{array}$ & $\begin{array}{l}\mathrm{Mn} \\
\mu \mathrm{g} / \mathrm{L}\end{array}$ \\
\hline & Iraqi Spec. & 10 & - & 0.7 & 10 & 3 & 1000 & 20 & 50 & 100 \\
\hline & WHO Spec. & 10 & 0.003 & 0.3 & 10 & 5 & 2 & 20 & 50 & 40 \\
\hline & Europian Spec. & 10 & 0.001 & 0.7 & 10 & 3 & 1 & 20 & 50 & 50 \\
\hline 1 & Utaifiya, Q409 & $<20$ & 0.11 & $<0.1$ & $<10$ & $<1$ & $<5$ & $<20$ & $<5$ & 1 \\
\hline 2 & Jihad, Q889 & $<20$ & $<0.1$ & $<0.1$ & $<10$ & $<1$ & $<5$ & $<20$ & $<5$ & 3 \\
\hline 3 & Zufaraniya, Q954 & $<20$ & 0.11 & $<0.1$ & $<10$ & $<1$ & $<5$ & $<20$ & $<5$ & $<1$ \\
\hline 4 & Mamudiya, Q 14 Ramdn & $<20$ & 0.21 & $<0.1$ & $<10$ & $<1$ & $<5$ & $<20$ & $<5$ & 1 \\
\hline 5 & Talbiya, Q 323 & $<20$ & 0.12 & $<0.1$ & $<10$ & $<1$ & $<5$ & $<20$ & $<5$ & $<1$ \\
\hline 6 & Mamudiya Q 17 july & $<20$ & 0.23 & $<0.1$ & $<10$ & $<1$ & $<5$ & $<20$ & $<5$ & $<1$ \\
\hline 7 & Kadhmiya, Q402 & $<20$ & 0.11 & $<0.1$ & $<10$ & $<1$ & $<5$ & $<20$ & $<5$ & 2 \\
\hline 8 & Shaab, Q337 & $<20$ & 0.12 & $<0.1$ & $<10$ & $<1$ & $<5$ & $<20$ & $<5$ & 2 \\
\hline 9 & 2n Amin, 741 & $<20$ & 0.12 & $<0.1$ & $<10$ & $<1$ & $<5$ & $<20$ & $<5$ & 1 \\
\hline 10 & Qarrada, Q905 & $<20$ & 0.12 & $<0.1$ & $<10$ & $<1$ & $<5$ & $<20$ & $<5$ & $<1$ \\
\hline 11 & Qarrada, Q905 & $<20$ & 0.16 & $<0.1$ & $<10$ & $<1$ & $<5$ & $<20$ & $<5$ & $<10$ \\
\hline 12 & Qadsiya, Q602 & $<20$ & 0.10 & $<0.1$ & $<10$ & $<1$ & $<5$ & $<20$ & $<5$ & 2 \\
\hline 13 & Salhiya, Q 28 Niss & $<20$ & 0.11 & $<0.1$ & $<10$ & $<1$ & $<5$ & $<20$ & $<5$ & $<1$ \\
\hline 14 & Falstin st, Q503 & $<20$ & 0.11 & $<0.1$ & $<10$ & $<1$ & $<5$ & $<20$ & $<5$ & $<1$ \\
\hline 15 & adr City, Q529 & $<20$ & 0.12 & $<0.1$ & $<10$ & $<1$ & $<5$ & $<20$ & $<5$ & $<1$ \\
\hline 16 & Dora Project (R) & $<20$ & $<0.1$ & $<0.1$ & $<10$ & $<1$ & $<5$ & $<20$ & $<5$ & 3 \\
\hline 17 & Dora Project (T) & $<20$ & $<0.1$ & $<0.1$ & $<10$ & $<1$ & $<5$ & $<20$ & $<5$ & $<1$ \\
\hline 18 & Whda Project (R) & $<20$ & $<0.1$ & $<0.1$ & $<10$ & $<1$ & $<5$ & $<20$ & $<5$ & 5 \\
\hline 19 & Whda Project (T) & $<20$ & $<0.1$ & $<0.1$ & $<10$ & $<1$ & $<5$ & $<20$ & $<5$ & 1 \\
\hline 20 & 9 Apr Project (R) & $<20$ & $<0.1$ & $<0.1$ & $<10$ & $<1$ & $<5$ & $<20$ & $<5$ & 5 \\
\hline 21 & 9 Apr Project (T) & $<20$ & $<0.1$ & $<0.1$ & $<10$ & $<1$ & $<5$ & $<20$ & & $<1$ \\
\hline 22 & Qadsiya Project (R) & $<20$ & $<0.1$ & $<0.1$ & $<10$ & $<1$ & $<5$ & $<20$ & & 2 \\
\hline 23 & Qadsiya Project (T) & $<20$ & $<0.1$ & $<0.1$ & $<10$ & $<1$ & $<5$ & $<20$ & $<5$ & $<1$ \\
\hline 24 & Wathba Project(R) & $<20$ & $<0.1$ & $<0.1$ & $<10$ & $<1$ & $<5$ & $<20$ & $<5$ & 10 \\
\hline 25 & WathbaProject (T) & $<20$ & $<0.1$ & $<0.1$ & $<10$ & $<1$ & $<5$ & $<20$ & $<5$ & $<1$ \\
\hline 26 & Karama Project (R) & $<20$ & $<0.1$ & $<0.1$ & $<10$ & $<1$ & $<5$ & $<20$ & $<5$ & 9 \\
\hline 27 & Karama Project (T) & $<20$ & $<0.1$ & $<0.1$ & $<10$ & $<1$ & $<5$ & $<20$ & $<5$ & $<1$ \\
\hline
\end{tabular}

TABLE 7. THE CONCENTRATION OF TRIHALOMETHANES IN TREATED (T) AND UNTREATED (R) WATER SAMPLES (DW).

\begin{tabular}{|c|c|c|c|c|c|c|}
\hline No. & Location & $\begin{array}{l}\text { Chloroform } \\
\mu \mathrm{g} / \mathrm{I}\end{array}$ & $\begin{array}{l}\text { Bromo form } \\
\mu \mathrm{g} / \mathrm{I}\end{array}$ & $\begin{array}{l}\text { Chloro dibromo } \\
\text { methane } \mu \mathrm{g} / \mathrm{I}\end{array}$ & $\begin{array}{l}\text { Dichloro bromo } \\
\text { methane } \\
\mu \mathrm{g} / \mathrm{I}\end{array}$ & $\begin{array}{l}\text { Total trihalo } \\
\text { methanes } \\
\mu \mathrm{g} / \mathrm{l}\end{array}$ \\
\hline 1 & Utaifiya, Q409 & 7 & 19 & 30 & 16 & 75 \\
\hline 2 & Jihad, Q889 & 21 & 13 & 31 & 25 & 90 \\
\hline 3 & Zufaraniya, Q954 & $<1$ & $<1$ & $<1$ & $<1$ & $<1$ \\
\hline 4 & Mamudiya,Q-Ram. 14 & $<1$ & $<1$ & 2 & 2 & 3 \\
\hline 5 & Talbiya, Q 323 & 3 & 23 & 21 & 8 & 55 \\
\hline 6 & Mamudiya-Q-Jul 17 & $<1$ & $<1$ & $<1$ & $<1$ & $<1$ \\
\hline 7 & Kadhmiya, Q402 & 14 & 14 & 32 & 22 & 82 \\
\hline 8 & Shaab, Q337 & 14 & 19 & 34 & 22 & 89 \\
\hline 9 & 2n Amin, 741 & 22 & 15 & 34 & 26 & 97 \\
\hline 10 & Qarrada, Q905 & 4 & 1 & 3 & 2 & 10 \\
\hline 11 & Qarrada, Q905 & 11 & 11 & 24 & 16 & 62 \\
\hline 12 & Qadsiya, Q602 & 16 & 13 & 30 & 22 & 81 \\
\hline 13 & Salhiya, Q 28 Niss & 10 & 15 & 28 & 16 & 69 \\
\hline 14 & Falstin st, Q503 & 17 & 13 & 32 & 24 & 86 \\
\hline 15 & adr City, Q529 & 31 & 9 & 25 & 28 & 93 \\
\hline 16 & Dora Project (R) & $<1$ & $<1$ & $<1$ & $<1$ & $<1$ \\
\hline 17 & Dora Project (T) & 44 & 6 & 34 & 32 & 116 \\
\hline 18 & Whda Project (R) & $<1$ & $<1$ & $<1$ & $<1$ & $<1$ \\
\hline 19 & Whda Project $(\mathrm{T})$ & 26 & 5 & 29 & 26 & 86 \\
\hline
\end{tabular}


TABLE 7 CONT. THE CONCENTRATION OF TRIHALOMETHANES IN TREATED (T) AND UNTREATED (R) WATER SAMPLES (DW).

\begin{tabular}{|l|l|c|c|c|c|c|}
\hline 20 & 9 Apr Project (R) & $<1$ & $<1$ & $<1$ & $<1$ & $<1$ \\
\hline 21 & 9 Apr Project (T) & 9 & 11 & 30 & $<$ & 68 \\
\hline 22 & Qadsiya Project (R) & $<1$ & $<1$ & $<1$ & $<1$ & $<1$ \\
\hline 23 & Qadsiya Project (T) & 46 & 5 & 34 & 31 & 116 \\
\hline 24 & Wathba Project(R) & $<1$ & $<1$ & $<1$ & $<1$ & $<1$ \\
\hline 25 & WathbaProject (T) & 20 & 10 & 39 & $<1$ & 97 \\
\hline 26 & Project karama(R) & $<1$ & $<1$ & 41 & 28 & 91 \\
\hline 27 & Karama Project (T) & 14 & 14 & 42 & & \\
\hline
\end{tabular}

Water hardness: From our experience, the water hardness of the Baghdad aqueduct is around $30^{\circ} \mathrm{FH}$ (French Degree $-1^{\circ} \mathrm{FH}$ is $=$ $10 \mathrm{ppm}$ of calcium carbonate equivalent dissolved in water). As for the samples analysed last December, the water hardness is waving between 30 and $45^{\circ} \mathrm{FH}$. In theory this parameter should be more homogeneous but it is not influencing the properties of the drinking water. The only effect is on the cleaning performances during washing.

It is interesting that the hardness values for water samples taken in Turkey which is located upstream of Tigris River ranges between
50 - $300 \mathrm{mg} / \mathrm{L}$ (Soylak et al., 2002). When hardness was correlated with other parameters like calcium concentration a perfect correlation was obtained as shown in Fig. 2. Although magnesium compounds are essential participant in the total hardness its correlation coefficient was far less than that of calcium with hardness. The fact that calcium in most of the samples analysed was the major cation attributes for the excellent correlation of the total cations with hardness as in Fig. 3

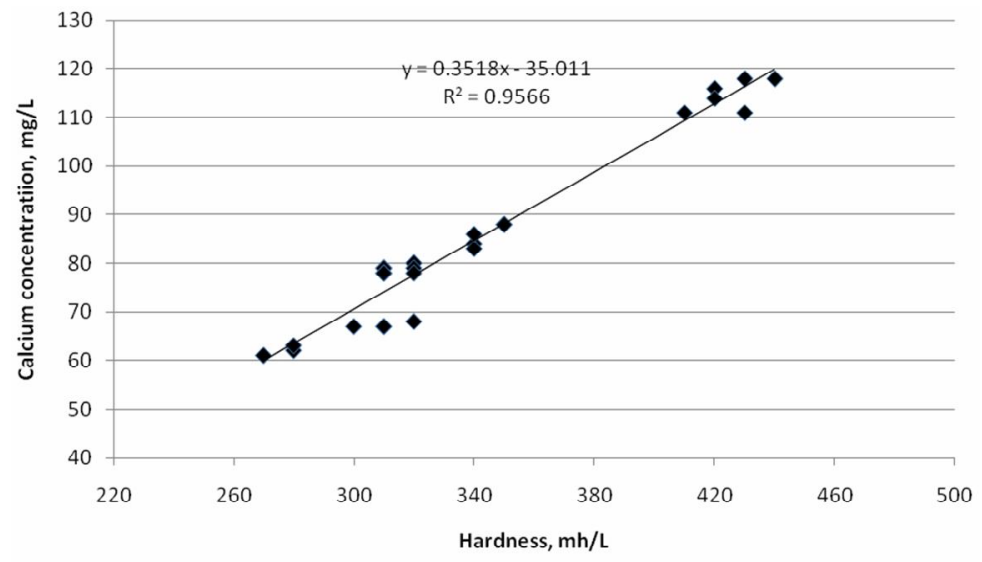

FIG. 2. CORRELATION OF HARDNESS WITH CALCIUM CONCENTRATION.

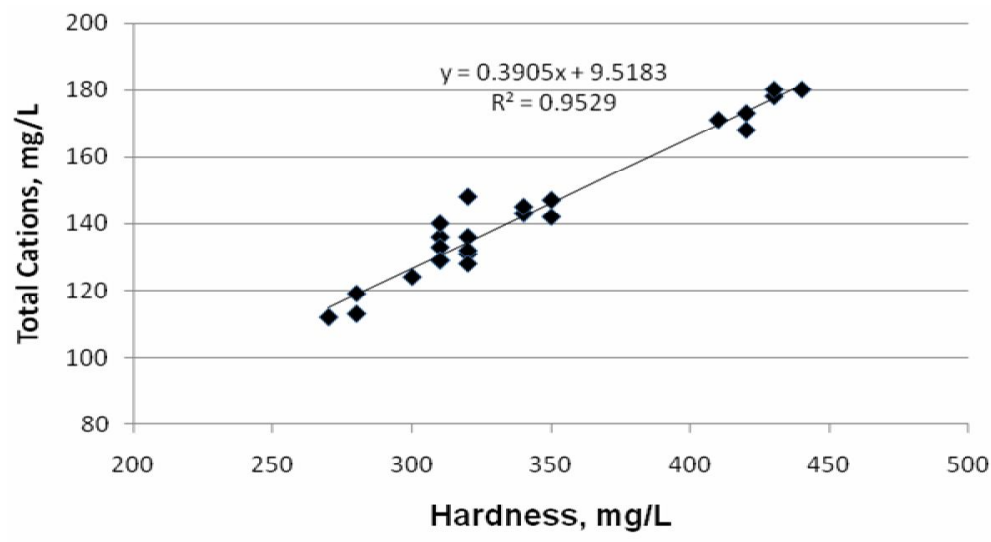

FIG. 3. CORRELATION OF HARDNESS WITH TOTAL MAJOR CATIONS. 
Electrical Conductivity (EC): The conductivity and TDS values fluctuated in the samples, being particularly high when sulphates and chlorides are present at high concentration. This could be due to possible chemical contamination during the water treatment directly in the aqueduct plant. The results of EC were correlated with TDS values for the analysed samples and the correlation coefficient was unity. Meanwhile, Fig. 4 shows reasonable correlation could be obtained with total major cations concentrations (0.81). With hardness values Fig. 5 shows that the correlation of EC values is reasonable (0.82).

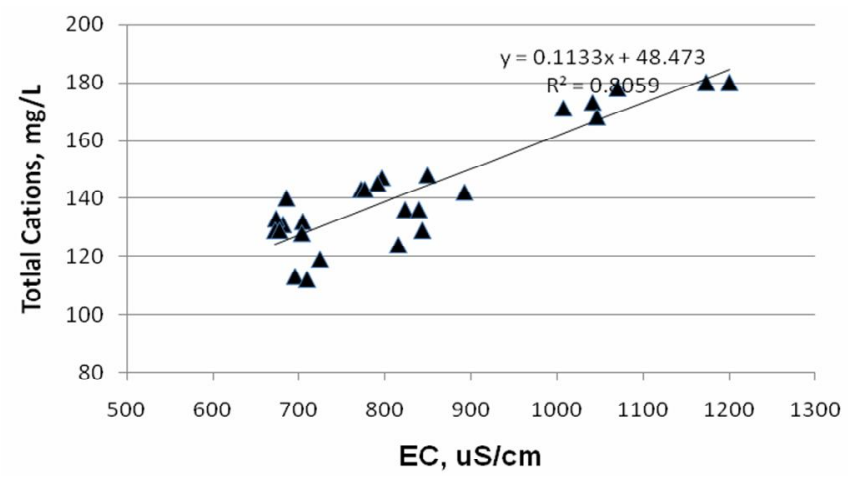

FIG. 4. CORRELATION OF EC VALUES WITH THE TOTAL MAJOR CATION CONCENTRATIONS.

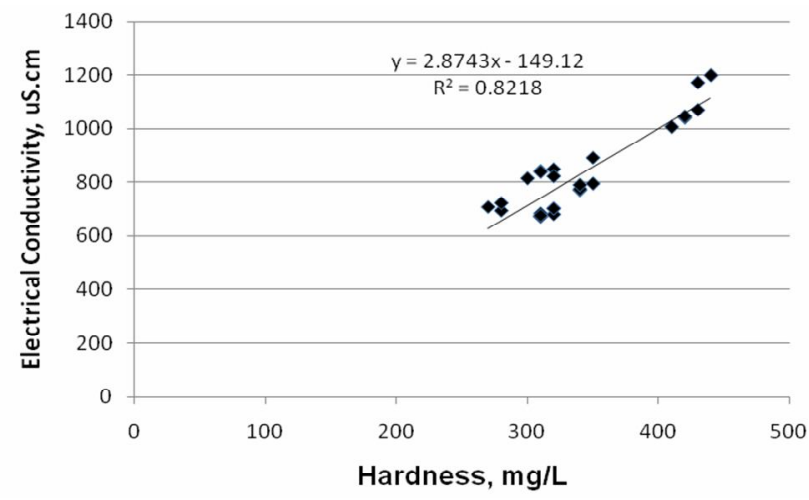

FIG. 5. CORRELATION OF EC VALUES WITH THE HARDNESS VALUES OF DW SAMPLES.

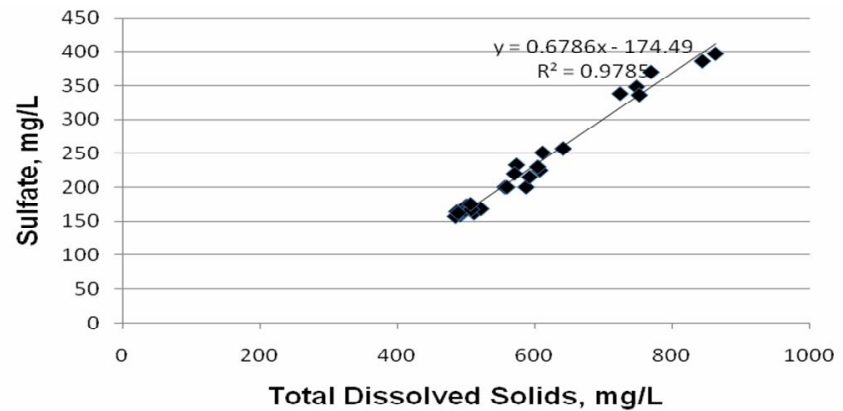

FIG. 6. CORRELATION OF SULFATE CONCENTRATION WITH TDS VALUES OF DW SAMPLES. 


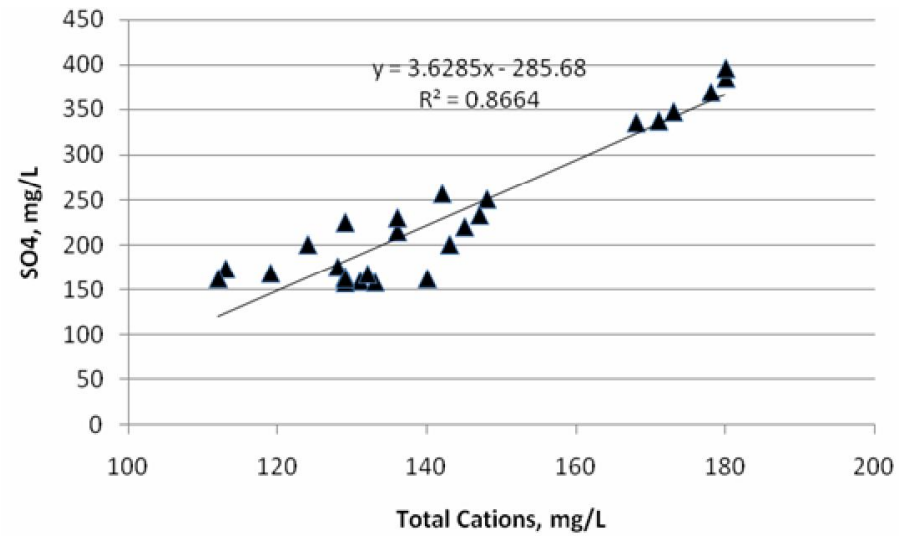

\section{FIG. 7. CORRELATION OF SULFATE CONCENTRATIONS WITH TOTAL CATION CONCENTRATIONS OF DW SAMPLES.}

The EC measurement of the samples indicated that the raw water and even after the treatment is characterized by relatively high and fluctuating EC values. They all fall between $600 \mu \mathrm{S} / \mathrm{cm}$ and 1200 $\mu \mathrm{S} / \mathrm{cm}$. The fluctuation is related to the season at which the sampling was made. The Iraqi standard does not include limits for the EC values of DW. Meanwhile, the WHO and the European standards recommended value of EC is $250 \mu \mathrm{S} / \mathrm{cm}$. This matter needs to be studied to correlate the EC values with human health and to manage for the introduction of EC limits within the Iraqi Standard. The increase in EC is related to the increase in chloride and sulfate ions.

With the exception of zinc, all residence DW samples exhibited metal ion concentrations that are low or within the accepted levels. However, the raw water samples showed relatively higher Fe (233$624 \mu \mathrm{g} / \mathrm{L})$ and $\mathrm{Al}(0.32-0.77 \mu \mathrm{g} / \mathrm{L})$ concentrations than the DW sample from the corresponding stations or the residence DW samples. For zinc there were four residence DW samples that have distinct high levels $(280-963 \mu \mathrm{g} / \mathrm{L})$ in comparison with the rest of the samples. These four locations were considered among the oldest neighbourhoods in Baghdad which is indicative of the effect of the old construction on the results. Even though, the zinc concentration in all of the analysed samples was within the acceptable values.

Sulphate: Sulphate appears as the predominant anion among the whole anions analysed and thus, it give excellent correlation coefficients with the main parameters of water quality, being 0.98 with TDS values (Fig. 6) and 0.87 with total cations (Fig. 7).

Looking at the micro elements detected with the analyses, we can imagine that the main flocculation of the raw water has been carried out with aluminium sulphate. The relatively high level of residual aluminium ions found should confirm this hypothesis. Probably, the raw materials have been dosed spot and not with a continuous plant. This could be the reason why a fluctuating concentration of certain elements has been found.

$\mathrm{Al}_{2}\left(\mathrm{SO}_{4}\right)_{3}+3 \mathrm{Ca}(\mathrm{OH})_{3} \rightarrow 2 \mathrm{Al}(\mathrm{OH})_{3}+3 \mathrm{CaSO}_{4}$

$\mathrm{Al}_{2}\left(\mathrm{SO}_{4}\right)_{3}+3 \mathrm{Ca}\left(\mathrm{HCO}_{3}\right)_{2} \rightarrow 2 \mathrm{Al}(\mathrm{OH})_{3}+3 \mathrm{CaSO}_{4}+6 \mathrm{CO}_{2}$

Reduction of sulphates, paying attention to the dosage of flocculants, playing with the $\mathrm{pH}$ and precipitating them with calcium hydroxide will reduce the conductivity and dry residue of the drinking water.
It is worthwhile to note that natural DW quality in some communities in Ghana, the sulphate levels do not exceed 8.02 $\mathrm{mg} / \mathrm{L}$ (Akoto \& Adiyiah, 2007. People that are not used to drinking water with high levels of sulphate can experience dehydration and diarrhoea. Babies are more sensitive to sulphate than adults. As a safety measure, water with a sulphate level exceeding $400 \mathrm{ppm}$ should not be used in the preparation of baby food (WHO directives). Older children and adults become used to high sulphate levels after a few days. In any case, water with high sulphates should be avoided for the rehydration of people with diarrhoea.

To confirm this hypothesis, it is necessary to carry out some checks on the raw water. Only these analyses will confirm the real origin of sulphates. Relatively high level of Magnesium, if compared with the Calcium content, should maintain the sulphate in solution. This is the reason why the addition of Calcium Hydroxide should help to remove the excess of sulphates. Clearly the addition of this chemical will reduce the efficiency of the plant. EU standards, in 1998, suggested a maximum of $250 \mathrm{mg} / \mathrm{l}$ of sulphates in water intended for human consumption.

Chlorides: In all the samples analysed, chlorides did not exceed the maximum limit. The EU max limit is $250 \mathrm{mg} / \mathrm{L}$. The chloride values on all the samples were in the range of $50-120 \mathrm{mg} / \mathrm{L}$. The real origin of the chlorides exceeding the $50 \mathrm{mg} / \mathrm{L}$ in this batch of samples is not fully understood. They waved from 50 to $120 \mathrm{mg} / \mathrm{L}$. This fluctuation is quite high if reported to the same origin of the raw water. As for sulphates, they should arrive from a contamination point just before the treatment plant or from the treatment process itself. In theory, chlorides could be originated from the chlorination process; in particular if the break point of the chlorine is very high but, in this case, the dosage of sodium hypochlorite should be very high (about $50 \mathrm{mg} / \mathrm{L}$ ) to destroy all the organic matter present in the raw water. This can justify an extra $70 \mathrm{mg} / \mathrm{L}$ of chlorides.

The use of degraded hypochlorite raw material used for water chlorination may account for a high concentration of chloride. For each mole of Hypochlorite a mole of sodium chloride is produced in the reaction. In general, sunlight and high temperature are the main causes of the Hypochlorite degradation. As by-product of the hypo degradation the formation of chloride occurs. See the reaction

$2 \mathrm{NaOCl} \rightarrow 2 \mathrm{NaCl}+\mathrm{O}_{2}$ 
In conclusion, degradation of the hypochlorite is the main cause of the chloride formation in the bleach used for the water treatment. Clearly, with a lower level of available chlorine in the bleach, a higher dosage is required to disinfect the water. As a consequence of this situation a very high dosage of chlorine may occur in the water treatment plant. As alternative to the sodium hypochlorite, a more stable but difficult to handle Calcium Hypochlorite could be used.

Boron: Boron contamination of water is impacting the male reproduction system of mammals (human included). For this reason, the EU legislation is now more restrictive. The maximum level allowed is $1 \mathrm{mg} / \mathrm{L}$ (WHO, 1998a). The origin of this element could be geogenic or from domestic detergents. However, boron compounds are rarely used in the production of detergents in Iraq. The boron content in nearly all the residence DW samples was higher than $0.1 \mathrm{mg} / \mathrm{L}$ which is only slightly higher than those of raw water samples. However, boron is widely distributed in surface water and groundwater. The average surface water concentration is about $0.1 \mathrm{mg} / \mathrm{L}$. In ground water boron concentrations in ground water can be as high as $10 \mathrm{mg} / \mathrm{L}$ in areas to the west of Euphrates River (Al-Dabbas, 2006). Besides, concentrations up to $0.4 \mathrm{mg} / \mathrm{L}$ have been found in most drinking water samples (Kwon, et al., 2005).

In this case boron could be only of natural origin. To exclude the sewage contamination two or three samples of the river water should be picked up to the north of the river; possibly outside the town. These samples should be compared with the samples picked up on the river just before the treatment plant.

Diaconu et al., (2008) reported boron concentrations levels of 0.02 $-1.55 \mathrm{mg} / \mathrm{L}$ in Romanian DW, which is comparatively higher than the results of Baghdad area DW.

Nitrogen compounds: No detectable ammonia and nitrite could be found in these DW samples. Generally they change their form when hypochlorite is added (Fair, et al. 1948. Ammonia becomes Chloroamine and Nitrite becomes Nitrate (Lahoutifard et al., 2003).

$$
\begin{aligned}
& \mathrm{NH}_{3}+\mathrm{HOCl} \rightarrow \mathrm{H}_{2} \mathrm{O}+\mathrm{NH}_{2} \mathrm{Cl} \text { (monochloramine) } \\
& \mathrm{NH}_{2} \mathrm{Cl}+\mathrm{HOCl} \rightarrow \mathrm{H}_{2} \mathrm{O}+\mathrm{NHCl}_{2} \text { (dichloramine) } \\
& \mathrm{NHCl}_{2}+\mathrm{HOCl} \rightarrow \mathrm{H}_{2} \mathrm{O}+\mathrm{NCl}_{3} \text { (trichloramine) }
\end{aligned}
$$

In any case, 3 of the samples received were not chlorinated (Al Zoufrania, Al Mahmodia and Mahmodia $17^{\text {th }}$ July). In all these samples, Ammonia and Nitrite are lower than the detectable limit. This means that, in general, we should not have a sewage contamination of the drinking water. The level of Nitrate is lower than that noticed in 2004 (Barbooti et al., 2005. This is probably due to the dry season with limited raining. Consequently, no surface water is available to wash away organic compounds in the river incoming water and all the Nitrogen available is fixed by the water plant present in the stream (Patriquin \& Knowles, 1972.

Iron: The various levels of iron in different samples of water could not be explained. The original $\mathrm{pH}$ of the river water is between 7.5 and 8.0. Corrosion of the pipes could partly explain this observation, because flocculation is seldom performed in Iraq by ferric chloride $\left(\mathrm{FeCl}_{3}\right)$. In any case, the relatively high levels of iron are not impacting the quality of the drinking water except the taste and the red residues on basins, showers, toilets, etc. High level of zinc on four samples can be explained with the probable corrosion of the pipelines. Zinc is not impacting the quality of drinking water.

Aluminum: The concentration of Aluminum in the analysed DW samples was in the range of $0.06-0.77 \mathrm{mg} / \mathrm{L}$. Aluminum levels in drinking-water vary according to the levels found in the source water and whether aluminum coagulants are used during water treatment (WHO, 1998b). In Germany, levels of aluminum in public water supplies averaged $0.01 \mathrm{mg} / \mathrm{L}$ in the western region, whereas levels in $2.7 \%$ of public supplies in the eastern region exceeded $0.2 \mathrm{mg} / \mathrm{L}$. In a 1993-1994 survey of public water supplies in Ontario, Canada, $75 \%$ of all average levels were less than 0.1 $\mathrm{mg} / \mathrm{L}$, with a range of $0.04-0.85 \mathrm{mg} / \mathrm{L}$. In a large monitoring program in 1991 in the United Kingdom, concentrations in 553 samples (0.7\%) exceeded $0.2 \mathrm{mg} / \mathrm{L}$ (Wilhelm \& Adel, 1995).

The fluoride concentration levels in all the analyzed DW samples were lower than the permissible levels. However, there exist some implications for the combined effects of aluminum with fluoride even at such low levels (Verner et al., 1998).

As for Sulphates, aluminium can be easily removed with Calcium Hydroxide. Detectable Aluminium presence in water at $\mathrm{pH}$ between 7.5 and 8.0 can be explained only with the flocculation process carried out with Aluminium Sulphate. Details are available in the Sulphate sections.

As for Sulphates, Aluminium can be easily removed with Calcium Hydroxide.

$\mathrm{Al}_{2}\left(\mathrm{SO}_{4}\right)_{3}+3 \mathrm{Ca}(\mathrm{OH})_{3} \rightarrow 2 \mathrm{Al}(\mathrm{OH})_{3}+3 \mathrm{CaSO}_{4}$

The products of this reaction $\left(\mathrm{Al}(\mathrm{OH})_{3}\right.$ and $\mathrm{CaSO}_{4}$ are insoluble in water. However, high water hardness should help this process and the addition of Calcium Hydroxide should not be necessary. The reaction of the calcium salts of water hardness with aluminium sulphate is reported below:

$\mathrm{Al}_{2}\left(\mathrm{SO}_{4}\right)_{3}+3 \mathrm{Ca}\left(\mathrm{HCO}_{3}\right)_{2} \rightarrow 2 \mathrm{Al}(\mathrm{OH})_{3}+3 \mathrm{CaSO}_{4}+6 \mathrm{CO}_{2}$

Trihalomethanes: The THM's formation in DW is a direct consequence of the chlorination process of the water with hypochlorite. Humic acids present in the ground water reacts with the chlorine forming these compounds. The maximum limit allowed by the EU legislation is of $30 \mathrm{ppb}$. These compounds are considered hepatotoxic and chloroform carcinogenic. The amount of total THMs allowed in drinking water is regulated by the USEPA, which has a set total THM annual average safe limit of $80 \mu \mathrm{g} / \mathrm{L}$ in drinking water (Tanglewood Water System, 2007).

Some studies linked THMs (Bromodichloromethane (BDCM), to possible stillbirths, miscarriages and birth defects, and bladder and rectal cancers. The Virginian Pilot reported that Chlorine mixes with organic materials, like algae and leaf particles, which includes chloroform and BDCM. In 1998, THM was suspected as a carcinogen and possible cause of miscarriages in Florida (Madabhushi, 1999). Around that time, a new water treatment plant was being upgraded and reports say the levels of THM were higher than normal for about nine months (Singer, 2006).

Except the sample picked from Al Zaafrania and the two samples picked from Al Mahmodia, all the water analysed contain THM's. This means that the water has been chlorinated properly to kill bacteria. Unfortunately the residual THM's present are over the EU limits. In any case, it must be noticed that WHO stated that it is better to have these contaminants in drinking water instead of bacteria. There are at least two techniques available to maintain these contaminants under the maximum limit. The first is the flocculation process and the second is the use of activated carbon. If necessary, both techniques can be used in series: flocculation first followed by filtration through activated carbon. 
Chlorine usually provides residual disinfection throughout the public-water distribution system. Ozone provides a residual disinfection for a limited time. However, bottled water may be in distribution for several weeks and storage conditions, especially temperature, may adversely affect quality. In terms of bacterial content, it is questionable as to whether bottled water is better than most municipal tap water (Kendall, 1992).

\section{CONCLUSIONS}

Generally, the samples of water analysed may be considered of good quality. Only 3 samples do not contain THM's. As we were sure of the chlorination of this water, the first consequence could be a microbiological contamination of this water with the related problems.

Organic contents of DW indicated the presence of organic materials. However, the flocculation with ferric chloride is considered better than Aluminium sulphate for the removal of humic acids. Iron is able to bind these organic compounds and remove them from the water. The relatively expensive adsorption on activated carbon is the most efficient process to remove these contaminants. It must be used after flocculation and the oxidation phase. However, it will completely remove the added chlorine which means that the DW must be chlorinated again with a low level of chlorine to maintain it free from bacteria in the pipelines during the distribution. The advantage of activated carbon is the ability to remove bad taste and smell from the water improving its organoleptic characteristics. Waterline plant in use at the Italian Red Cross hospital in the Medical City Centre, Baghdad, applies this type of process.

\section{REFERENCES}

Akoto, O., Adiyiah, J. (2007). Chemical analysis of drinking water from some communities in the Brong Ahafo region, International Journal of Environmental Science and Technology, 4: 211-214.

Al-Dabbas, M. (2006). Distribution of Boron in the Ground Water of Kerbala, western Iraq, M.Sc. Thesis, Geology Department., University of Baghdad, Iraq.

Alsudany, H. M. L.; Al-Bayati, R. I. \& Barbooti, M. M. (2009), Determination of anions by ion chromatography in water samples of Baghdad city, African Journal of Pure and Appl chemistry, 3. 165-169.

Barbooti, M. M.; Hatam, N.; Akram, B.; Joseph, F.; Ibraheem, S. \& Fadhil, A. (2005). "The Quality Of Drinking Water In Baghdad Area After-The War", Proceedings of the $1^{\text {st }}$ International Conference on Environmental Health in Iraq, Amman, 19-22.

Barbooti, M. M.; Jassem, I. A. \& Kadhum, R. (2006), Evaluation of DW purification units in Iraq, Report No. 261, Ministry of Environment, Iraq.

Diaconu, D.; Nastase, V.; Nănău, M. M., Nechifor, O. \& Nechifor, E., (2008). Estimation Of Boron Concentration In Some Drinking Water Samples Of Rural Areas. Journal of Preventive Medicine, 16: $77-84$.

Duruibe, J. O. Ogwuegbu, M. O. C. \& Egwurugwu, J. N., (2007). Heavy metal pollution and human biotoxic effects, International Journal of Physical Sciences, 2: 112-118.

Environmental Working Group, (2005). Chemical aspects, A National Assessment of Tap Water Quality, National Tap Water Quality Database, Dec. 20,

http://www.who.int/water sanitation health/dwq/gdwq3 12.pdf.
Fair, G. M., Morris, J. C. Chang, S. L. Weil, I. \& Burden, R. P. (1948). The behavior of chlorine as a water disinfectant. Journal of American Water Works Association, 40: 1051-1061.

Fawell, J.; Bailey, K.; Chilton, J.; Dahi, E.; Fewtrell, L. \& Magara, Y., (2006). Fluoride in Drinking-water, WHO, TJ International (Ltd), Cornwall, UK.

Kendall, P. (1992) Drinking Water Quality and Health, Food And Nutrition Series, No. 9.307, Colorado State University, Cooperative Extension. 10/92. www.colostate.edu/Depts/CoopExt.

Kwon, T.; Hirata, M.; Sakuma, S.; Hano, T \& Yamagishi, T. (2005), Continuous Recovery of Boron from Wastewater with a Dio, Solvent Extraction and Ion Exchange, 23: 391-400.

Lahoutifard, N.; Lagrange P. \& Lagrange, J. (2003), Kinetics and mechanism of nitrite oxidation by hypochlorous acid in the aqueous phase. Chemosphere, 50: 1349-1357.

Madabhushi, B. S. (1999). What are trihalomethanes?, http://www.nesc.wwu. edu/ndwc/articles/QandA/OTsp99_Q_A.pdf

NSF International, (2000). Review of Contaminant Occurrences in Drinking Water Treatment Chemicals, Report, Prepared for Health Canada, Health Protection Branch, March 312000.

Patriquin, D. \& Knowles, R. (1972). Nitrogen fixation in the rhizosphere of marine angiosperms, Marine Biology, 16: 49-58.

Self, J. R. (1996). Domestic Water Quality Criteria, Crop Series, Soil, Report 0.513, Colorado State University Cooperative Extension. 4/96.

Singer, P. C. (2006). Disinfection Byproducts in Drinking Water: Additional Science and Policy Considerations in the Pursuit of Public Health Protection, National Water Research Institute, The Clarke Lecture.

Soylak, M.; Armagan Aydin, F.; Saracoglu, S.; Elci, L. \& Dogan, M. (2002). Chemical Analysis of Drinking Water Samples from Yozgat, Turkey, Polish Journal of Environmental Studies, 11: 151156

Squillqace, J.; Scott, J. C.; Moran, M. J.; Nolan, B. T. \& Kolpin, D. W., (2002). VOCs, Pesticides, Nitrate, And Their Mixtures In Groundwater Used For Drinking Water In The United States. Environmental Science and Technology, 36: 1923-1930.

Tanglewood Water System, Water Quality Report, (2007), Golden State Water Company, http://www.ewg.org/tapwater/yourwater/ system. . php? Pwsid =TX1910013

Verner, J. A.; Jensen, K. F.; Horvath, W. \& Isaacson, R. L. (1998). Chronic administration of aluminum-fluoride or sodium-fluoride to rats in drinking water: alterations in neuronal and cerebrovascular integrity. Brain Research, 784

Vodela, J. K.; Lenz, S. D.; Renden, J. A.; Mcelhenney, W. H. \& Kemppainen, B. W. (1997). Drinking water contaminants (arsenic, cadmium, lead, benzene, and trichloroethylene). 1. Interaction of contaminants with nutritional status on general performance and immune function in broiler chickens. Poultry Science, 76:14741492.

Water Supply Division, (2005), Drinking Water Standards Governing Drinking Water Quality and Reporting Requirements for Public Water Systems, 30 TAC Chapter 290 Subchapter F, RG346 (Revised), Texas Comm. Environ. Qual.,April, www.tceq.state. tx.us/publications. 
World Health Organization WHO, (2005), "Trihalomethanes in Drinking-water", Background document for development of WHO Guidelines for Drinking -water Quality, 05.08/64.

Water Pollution Control Regulation of Turkish Authorities (WPCRTA) (Su Kirliligi Kontrol Yonetmeligi), (1989), No:19919, T.C. Resmi Gazete.

World Health Organization (WHO), (2004), Guidelines for Drinking Water Quality, Chap. 8, Geneva.

World Health Organization (WHO), (1998a). Guidelines for drinking-water quality- Boron, 2nd ed. Addendum to Vol. 2. Health criteria and other supporting information. World Health Organization, Geneva.
World Health Organization (WHO), (1998b). Guidelines for drinking-water quality- Aluminum", 2nd ed. Addendum to Vol. 2. Health criteria and other supporting information. World Health Organization, Geneva.

Wilhelm, M. \& Adel, H., (1995). Aluminum in drinking water: a risk factor for Alzheimer's disease?, Forum, Städte, Hygiene, 46: 255258. 